\title{
The Technology Explorers: Partnering with Older Adults to Engage with Virtual Reality and Virtual Avatars
}

\author{
Authors \\ Steven Baker, The University of Melbourne \\ Jenny Waycott, The University of Melbourne \\ Frank Vetere, The University of Melbourne \\ Thuong Hoang, Deakin University
}

\begin{abstract}
Participatory research methods are increasingly being adopted when designing and evaluating emerging technologies for older adults. This chapter details our approach to applying participatory methods to our work with a group of older adults during series of workshops. The workshops involved working with 25 adults from both regional and metropolitan areas, who were over the age of 70. We referred to these participants as the Technology Explorers. The Technology Explorers partnered with us to explore how Virtual Reality (VR) technology could be harnessed to provide older adults with opportunities to participate in meaningful social activities. This chapter focusses on the initial engagement phase of the project that involved introducing the Technology Explorers to a range of VR systems and interaction techniques, co-designing 3D Avatars, and interacting with each other in two social VR technology probes. We describe the participatory methods that helped us to build a strong collaborative partnership with the Technology Explorers, before detailing the techniques used to engage with group members who had vastly different levels on technical knowledge. As none of the Technology Explorers had any experience with VR or virtual avatars, in a final section we describe three techniques we employed to develop this capacity in the group, with a particular focus on the deployment of two technology probes that marked the end of the workshop series.
\end{abstract}

\section{Keywords}

Virtual Reality; Older Adults; Avatars; Action Research; Technology Probes

\section{Highlights}

- Participatory engagement with older adults offers unique advantages when exploring cutting-edge technologies. 
- Participatory methods allow researchers to iteratively build technical knowledge within groups with varying capabilities.

- Older adults bring a unique perspective to technology evaluation which helped us better understand the challenges and opportunities present in developing social VR applications. 


\subsection{Introduction}

In this chapter, we discuss our experiences partnering with 25 older adults aged 70 and over, who we refer to as the Technology Explorers. The Technology Explorers are participants in an Australian Research Council (ARC) funded research project that aims to investigate the role Virtual Reality (VR) technology and virtual avatars can play in fostering social participation between groups of older adults. The focus of this chapter is the initial exploratory stage of the project that involved a series of workshops in regional and metropolitan Victoria, Australia. Our experiences with the Technology Explorers provide a case study example of how participatory methods helped the Technology Explorers to develop as a cohesive group, address the differing levels of technical expertise within the group, and build knowledge about VR and virtual avatars.

The inclusion of both regional and urban older adults was important to help us meet the broader goals of the project - that is, to examine ways of using new technologies for social enrichment in old age. In Australia, the experiences of ageing communities in rural and regional locations, and their access and adaption to emerging technologies, are of particular interest to researchers and policy-makers (Baker, Warburton, Hodgkin, \& Pascal, 2017; Warburton, 2014; Warburton, Cowan, Winterton, \& Hodgkin, 2014; Winterton \& Warburton, 2011). Opportunities for social participation, and access to services, can be particularly scarce in these locations. We therefore aimed to engage both regional and urban communities in exploring and designing social VR experiences for older adults.

The chapter is arranged in three sections. In the first, we provide a brief overview of the existing literature on older adults' use of VR technologies and avatars to help set the context for the workshop series. Section two provides a summary of the methodology and methods underpinning the study that laid a firm methodological foundation for achieving our participatory goals and thereby building a strong collaborative base for the study. In the third and final section, we discuss three separate participatory strategies that were employed over the first workshop series and provide some observations on how these strategies supported meaningful engagement with the social VR technology probes that concluded this part of the study. We begin by providing examples of a number of general participatory techniques, drawn from action research methods, that helped to build a strong group culture for both the Technology Explorers and the researchers involved in the workshops. We then describe a number of specific techniques that were adopted to address the disparity in technological 
knowledge that was evident within the Technology Explorer groups. Finally, we discuss the specific techniques that were employed to engage the Technology Explorers in a dialogue about how the emerging technology of social VR and avatars might be best applied to benefit older users and encourage social participation in virtual worlds. This includes a discussion of the two technology probes that were deployed in the final two workshops and how these probes helped to elicit concrete design priorities from the Technology Explorers with respect to social VR and virtual avatars.

\subsection{Research Context}

The project reported in this chapter builds on, and contributes to, a recent move in humancomputer interaction research towards developing technologies that provide social and hedonistic benefits for older adults. That is, technological advancement is no longer viewed as predominantly offering ways to support and help care for older adults. Instead, there is growing recognition that older adults use, and gain benefit from using, social technologies such as social media (Brewer \& Piper, 2016), photo-sharing applications (Waycott et al., 2013), YouTube (Harley \& Fitzpatrick, 2009), and games (Brand \& Todhunter, 2015). Alongside this move, research has also explored numerous creative ways of engaging older adults in the design process. These include playful approaches, such as "making" (Rogers et al., 2014), and providing design provocations to elicit reflections and ideas about technological innovation (Light, Howland, Hamilton, \& Harley, 2017).

\subsubsection{VR and Older Adults}

Despite these advancements, there is no extant research looking specifically at the use of VR technology with older users as a means of encouraging social participation. The overwhelming majority of VR studies instead conform to the "support and care" model of research. For example, research has explored the potential for VR technologies to be used for gait training, mitigation of falls risk and functional balance rehabilitation (Bisson, Contant, Sveistrup, \& Lajoie., 2007; Mirelman et al., 2011, 2013; Schultheis \& Rizzo, 2001). There has also been limited research examining the use of VR exergames (exercise games) with older adult users (Anderson-Hanley, Snyder, Nimon, \& Arciero, 2011; Cyarto, Batchelor, Baker, \& Dow, 2016; Miller et al., 2014), however, social interaction in these interventions has been secondary to the movement based goals of the designs.

There also remains a lack of technical clarity about what exactly constitutes a VR system, especially as it relates to the viewing experience, in some designs aimed at older users. For 
example, there is a small but growing body of research focusing on the application of VR for residents with dementia (Flynn et al., 2003; Moyle, Jones, Dwan, \& Petrovich, 2017). However, these studies have used screen projected, rather than fully immersive, VR models. In this chapter, a VR system is defined as one that provides a fully immersive experience, where the user's field of vision is completely covered by the use of VR headset or headmounted displays. Furthermore, previous interventions to date have focused solely on individual interactions with a virtual environment, rather than exploring group social interactions with VR technology, which is one of the aims of the project discussed in this chapter.

\subsubsection{Older Adults and Virtual Avatars}

As part of our work to explore the role of VR in active ageing, we needed to understand older adults' choices in relation to the avatars they would choose to use in a virtual environment and what these choices might reveal about their views on the ageing body and selfrepresentation. This was crucial, as in order to interact and have agency in a virtual world, the user must be embodied as an avatar. As with the VR literature, there is relatively little research examining older adults use of avatars in fully immersive virtual environments. However, a body of research does exist examining older adults and avatars in $2 \mathrm{D}$ virtual worlds.

A number of research projects have investigated the use of avatars as virtual assistants. A succession of studies in this area suggests that older adults, including those with mild cognitive impairment, react positively to the avatar virtual assistant (Carrasco et al., 2008; Castilla et al., 2013; Singh, Johnson, Alnizami, \& Gilbert, 2011; Zancanaro et al., 2013). A smaller body of literature has examined older adults' preferences in creating or choosing avatars. In an exploratory study that was explicitly focused on evaluating older adults avatar preferences, Cheong, Jung and Theng (2011), asked 24 older adults (over the age of 55) to evaluate 20 custom-made $2 \mathrm{D}$ avatars. Results suggest a preference for child-like avatars and animal or object forms (Cheong et al., 2011). Cyarto, Batchelor, Baker and Dow (2016), evaluated an exergame system used to conduct a virtual exercise program with older adults (women aged 64-74). Although the research did not have a specific focus on avatar creation, participants noted that the construction and use of avatars was one of the most enjoyable aspects of the project. 


\subsection{Methodology and Methods}

\subsubsection{Action Research}

A key goal of the project was to facilitate active participation in the research process by the Technology Explorers. Therefore, we needed to choose a methodological approach that provided strategies for strengthening engagement with our participants.

Action research offered a clearly articulated set of methods that we believed would serve to foster active participation by the Technology Explorers. According to Reason and Bradbury (2008, pp. 3-4), action research:

- Is a set of practices that responds to people's desire to act creatively in the face of practical and often pressing issues in their lives within organizations and communities;

- Calls for engagement with people in collaborative relationships, opening new 'communicative spaces' in which dialogue and development can flourish;

- Draws on many ways of knowing, both in the evidence that is generated in inquiry and its expression in diverse forms of presentation as we share learning with wider audiences;

- Is value oriented, seeking to address issues of significance concerning the flourishing of human persons, their communities, and the wider ecology in which we participate;

- Is a living, emergent process that cannot be predetermined but changes and develops as those engaged deepen their understanding of the issues to be addressed and develop their capacity as co-inquirers both individually and collectively.

These properties succinctly articulate our desired approach for the research project. Another benefit of the action research approach is its problem-solving process that incorporates cycles of planning, action and reflection throughout all phases of the research process, a technique that can be traced back to the work of Kurt Lewin (Brydon-Miller, Greenwood, \& Maguire, 2003; Lewin, 1946; Ozanne \& Saatcioglu, 2008; Reason \& Bradbury, 2008; Zuber-Skerritt \& Perry, 2002). This problem-solving process was ideally suited to the monthly workshop schedule that we adopted for the project. Figure 0.1 illustrates how these cycles were incorporated into this study is included below. 


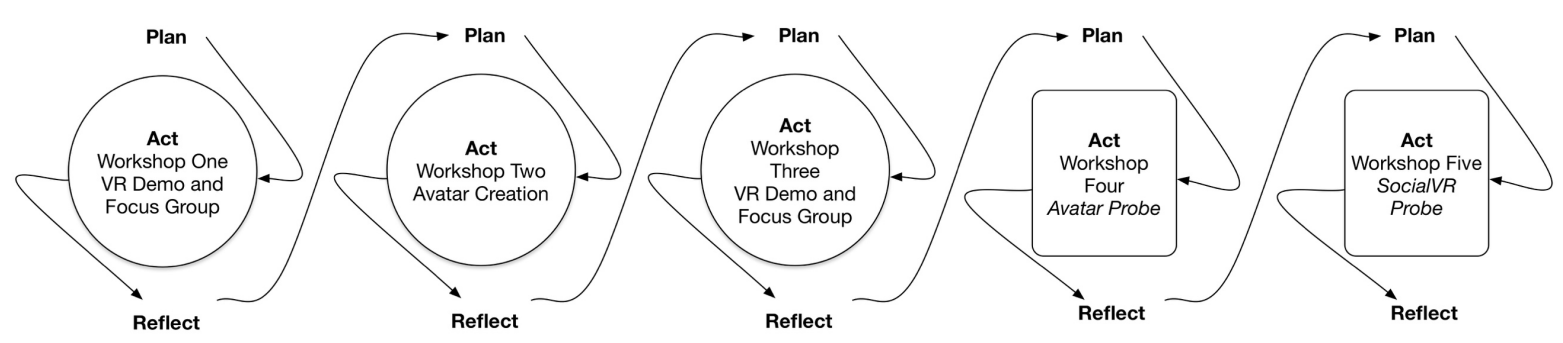

Figure 0.1: Action Research Cycles of Workshop Series.

\subsubsection{Technology Probes}

In order to provoke discussion about the specific design challenges that would need to be addressed in order to engage with social VR applications, the first workshop series concluded with the deployment of two technology probes. Technology probes aim to skilfully balance three different disciplinary goals. Hutchinson et al. (2003), describe these as; "the social science goal of collecting information about the use and the users of the technology in a realworld setting, the engineering goal of field-testing the technology, and the design goal of inspiring users and designers to think of new kinds of technology to support their needs and desires" (p. 18). Technology probes have been used extensively in the field of Human Computer Interaction (HCI) and have been successfully deployed in projects with older adults (Vutborg, Kjeldskov, Pedell, \& Vetere, 2010). We deployed two technology probes, the Avatar Probe and the SocialVR Probe. Both technology probes incorporated avatars designed by the Technology Explorers in an early workshop.

\subsection{Participatory Engagement with the Technology Explorers}

A survey conducted prior to the first Technology Explorer workshop, revealed some interesting information about the technological literacy of the Technology Explorer groups. Rather than most participants having only a general level of technological experience, the Technology Explorer groups consisted of wildly diverse experience levels ranging from participants with limited to no previous experience with computers, through to expert users with decades of experience. The one consistent result from the questionnaire, however, was that neither group had experience with VR technology.

While these findings are consistent with recent research that has emphasised the heterogeneous nature of older adult groups with respect to technological knowledge (Baker, Waycott, Pedell, Hoang, \& Ozanne, 2016; Hill, Betts, \& Gardner, 2015; Vines, Pritchard, Wright, Olivier, \& Brittain, 2015; Waycott et al., 2016), we were none-the-less faced with the 
challenge of how best to engage with such a diverse group on technical matters relating to the workshops. In this section, we first discuss some of the techniques, derived from action research principals that we used to build a strong group culture within the Technology Explorer groups. We then describe the various methods employed to address the diverse levels of technical knowledge that were evident in the groups. To conclude the section, we detail three techniques that were employed to help the Technology Explorers gain a better understanding of social VR technology and embodiment as a virtual avatar. Pseudonyms, chosen by the Technology Explorers at the beginning of the research project, are used when describing their responses to some of the measures employed during the workshop series.

\subsubsection{Participatory Engagement Techniques}

In order to build a strong participatory culture within the Technology Explorer groups, we drew heavily on our methodological choices to help guide our action. Core action research principles clearly called on us to engage all of the Technology Explorers in the project on a technical level, to embrace the diverse ways of knowing in the group, help the Technology Explorers to deepen their understanding, and develop their capacities both individually and collectively.

A first step to achieving this goal was to employ a number of participatory measures to build group confidence and cohesion. A measure that proved very valuable in our experience was the incorporation of a casual, purely social, element into each monthly workshop. This involved both researchers and participants sharing a communal lunch. During this time, researchers and participants were given the opportunity to have informal discussions and gain a better understanding of each other as individuals outside our roles in the study. This was also an important means by which power relationships were challenged by engaging within a more egalitarian space where life experience and social skills were privileged above formal qualifications or technical knowledge. This informal and communal aspect of the workshops, in combination with other methods such as; adopting a relatively long data collection period (December 2016 to May 2017) and incorporating a number of 'ice-breaker' exercises into the workshop program, proved crucial in ensuring that the Technology Explorers groups developed a cohesive and supportive group culture.

These participatory techniques proved very valuable over the course of the initial workshop series. Many comments from the Technology Explorers singled out these elements of the 
workshops as being one of the reasons they remained engaged and excited about the work ahead.

\subsubsection{Engaging Older Adults with Diverse Technical Knowledge}

While the participatory measures highlighted above were important, they did not directly address the diverse levels of technical knowledge across the Technology Explorer groups. It was vital that techniques be developed to support those with less technical knowledge to grow in confidence. Similarly, care needed to be taken to ensure that those with high levels of technical skill were encouraged to draw on this knowledge to better understand VR technology. Two specific measures that proved valuable here were providing opportunities for smaller sub-groups to develop within the workshops to access technical knowledge, and building a shared experience based on the fact that all group members lacked knowledge about VR technology and virtual avatars.

To allow space for sub-groups to develop that could address different levels of technical understanding, additional members of the research team, over and above those already conducting the workshop, attended each workshop session. This provided opportunities for more interpersonal interaction and gave individual participants the ability to communicate within smaller sub-groups rather than having to always contribute to the larger group. This allowed informal spaces to organically emerge, where participants could seek further information, whether that be more in-depth technical knowledge, or to ask a question that a participant might feel was too 'basic' for the larger group. A good example of this technique in practice occurred in the first metropolitan workshop. During the first VR demonstrations, a number of more technically advanced members of the group were intrigued as to how VR systems differed technically from other ICTs. The presence of additional members of the research team meant that someone with expert technical knowledge about VR development was on hand to convene an impromptu sub-group discussion with these Technology Explorers, while others continued to watch the demonstration and engaged in a more general discussion of VR.

While the application of this technique helped us to engage with the Technology Explorers across various levels of technological understanding, it was important for the overall goals of the project that we developed a cohesive and united group culture in which everyone felt that they were able to make a valuable contribution. Our solution was to leverage the fact that although there were differing levels of technical understanding within the group in relation to 
traditional desktop and touch-screen forms of ICT, all the Technology Explorers lacked specific knowledge of avatars and VR. This provided us with a means of building solidarity around the groups exploration of VR regardless of their levels of expertise with other forms of technology. In the next section, we detail some of the methods we applied during the workshops to engage the Technology Explorers in an exploration of avatars and VR technology.

\subsubsection{Developing the Technology Explorers Knowledge of VR and Avatars}

One of the techniques that proved valuable was to incorporate demonstrations of a range of existing VR hardware and software into the workshop series. Two of the first three workshops involved engaging with a range of VR systems and trying various VR software. The goal here was to introduce the Technology Explorers to a range of interaction methods and approaches to VR that were possible. This ranged from simple technology for viewing 3D environments, such as Google Cardboard, through to more advanced systems such as the Oculus Rift and Microsoft Hololens that allowed the Technology Explorers to manipulate 3D objects in virtual environments. This diverse engagement with a range of VR tools helped to build a broad conception of what was possible in VR within the groups. As all the Technology Explorers shared in the experience of using VR for the first time, all were able to share their thoughts and questions safe in the knowledge that they were all equally qualified to discuss VR.

As another key goal of the project was to build knowledge about virtual avatars in the group, the second workshop consisted of a guided workshop using an open source 3D avatar creation tool called MakeHuman (www.makehuman.org) to create a personal avatar for each participant. During these workshops, we again employed the technique of having additional 'roving' members of the research team to allow individuals with poorer technical skills to seek additional help and to allow those with advanced skills to seek additional knowledge about the software. An added benefit of these sessions was that the resulting avatars, designed by each Technology Explorer, were then able to be imported and used in the technology probes that would conclude the workshop series. While the overwhelming majority of the Technology Explorers enjoyed the avatar creation workshop (as measured by the responses to a post-workshop questionnaire), there were a small number of participants for whom the expectation to use quite complex desktop software, especially so early in the research project, proved daunting. This illustrates one disadvantage of incorporating the technology probes into the workshop series. The decision to hold the avatar creation 
workshop so early in the workshop series was a direct result of the need to allow time to incorporate the resulting avatars into the technology probes. It did not, however, allow sufficient time to build skills and confidence within the group about using the MakeHuman software.

A final method by which the Technology Explorers were encouraged to build a strong collaborative culture by engaging with VR technology involved the deployment of the two technology probes in the final two workshops of the series. Technology probes evolved from earlier cultural probe work by Gaver and colleagues (Gaver, Boucher, Pennington, \& Walker, 2004; Gaver \& Dunne, 1999). As with cultural probes, technology probes are designed to be provocations to help elicit new perspectives on design. The first of our probes, the Avatar Probe involved the groups coming face-to-face with, and embodying, a life size version of the avatar they created in the second workshop. These avatars were projected onto a screen in front of the Technology Explorers and they were able to control the movement of the avatar via Microsoft Kinect 3D cameras that mapped the movement of the Technology Explorer to their avatar. A screen shot of Technology Explorers interacting with the Avatar Probe is shown in Figure 0.2 below.

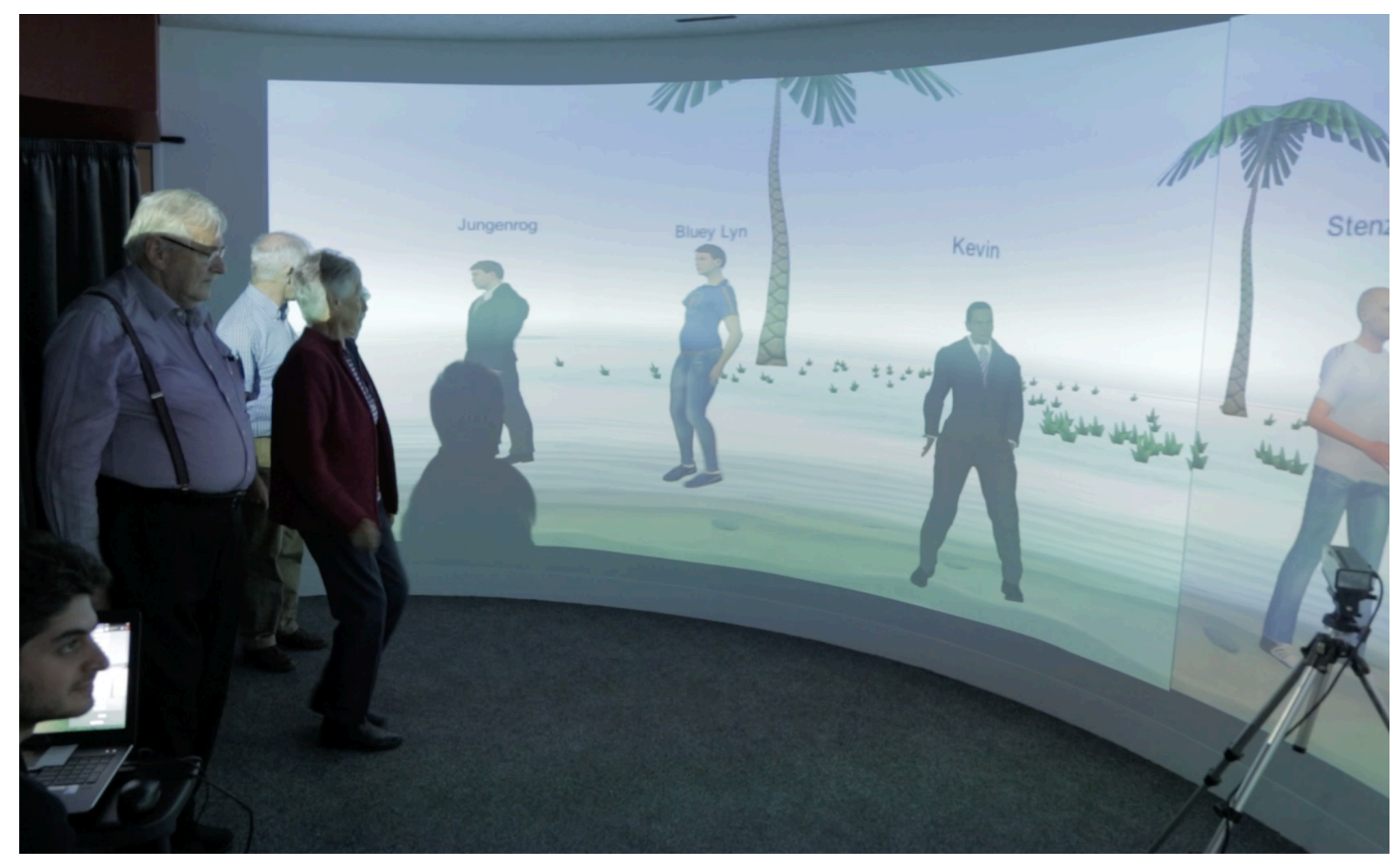

Figure 0.2: Technology Explorers Interacting with the Avatar Probe. 
The second technology probe, the SocialVR Probe, provided an opportunity for the Technology Explorers to enter a fully immersive virtual world as their avatar and participate in a group conversation with other Technology Explorers who were located in separate rooms around a university campus. A key difference between the Avatar Probe and the SocialVR Probe was that the former involved viewing the avatar in a third person perspective, whereas the latter involved being embodied as an avatar in the first person. An image showing a screenshot of the SocialVR Probe is shown in Figure 0.3.

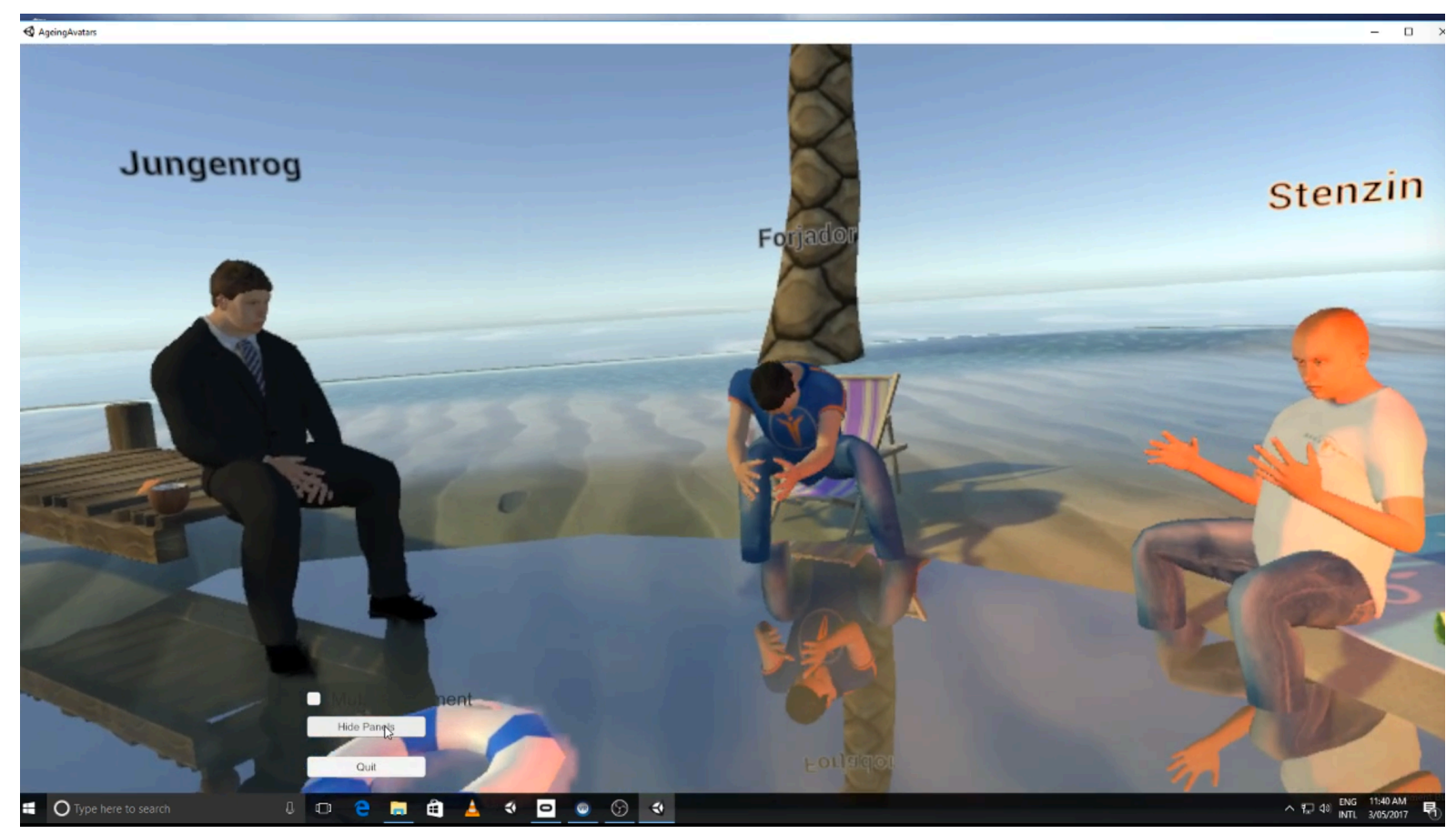

Figure 0.3: Screenshot of the SocialVR Probe.

The deployment of the technology probes proved to be a valuable way to conclude the workshop series and served as a bridge between the exploratory and participatory design stages of the project. Two key benefits of the technology probes were their ability to provide the Technology Explorers with an early experience of a bespoke social VR application, and to stimulate more specific discussion of the Technology Explorers design priorities for social VR.

While the early workshops introduced the Technology Explorers to a range of VR systems and software, it was only at the technology probe stage of the workshops that some of the fundamental elements of social VR systems were explicitly introduced. Core social VR concepts such as the use of full body avatars, body tracking via 3D cameras, and 3D audio were first introduced to the Technology Explorers in the technology probe workshops. The 
technology probes also introduced the Technology Explorers to a bespoke rather than commercial VR implementation. This was helpful in regards to setting expectations about the focus of the next stage of the research project, particularly in relation to the design focus on social participation and interaction in virtual worlds.

\subsubsection{Observations from the Technology Explorers Engagement with the Technology Probes}

The engagement with the technology probes produced a marked change in the way the Technology Explorers conceptualized VR. Whereas comments in the early stage of the project focused on how VR might be valuable for 'other' older adults, such as those with physical disabilities and those living in residential aged care facilities, engagement with the probes provoked the Technology Explorers to consider what was important to them as individuals when using social VR. This change often manifested as a more critical engagement with the probes, especially in relation to the realism of the avatars. In particular, engagement with the technology probes highlighted the importance of facial expression in the avatars for the Technology Explorers.

Earlier research into older adults use of avatars has shown characteristics such as youthfulness to be those most valued by older users (Cheong et al., 2011; Rice et al., 2013). When engaging in a social interaction using the probes, however, the Technology Explorers overwhelmingly focused on the limited ability of their avatars to show emotion. This led to them comparing their avatars to "dolls or marionettes" (Tina and Gordon) or "zombies" (Herb and Samuel). This focus on the importance of facial expression for the Technology Explorers was perhaps best expressed by Violet who, after engaging with the probes, commented "One thing I would love to be able to change is the expression on the face. Have a more smiley one. Because if you are interacting with someone, you want to be smiling."

Engagement with the probes also highlighted the importance of accurate body tracking to the Technology Explorers. A characteristic of the Microsoft Kinect 3D cameras that were used to translate the Technology Explorers' movements to their avatars in the probes was that occasional tracking errors would cause the avatars to move in unpredictable, and very nonhuman ways. Rather than this proving to be a slight annoyance, many Technology Explorers highlighted how these errors had the effect of disrupting their feeling of being immersed in the virtual world. Some even described their avatars as having "Parkinson's disease" (Bob), with reference to the jittering of the avatar hand movements due to the sensor errors of the Kinect. 
While the section above illustrates the benefits the technology probes had in eliciting more concrete and personal views about the use of social VR and avatars, the use of technology probes was also problematic in one respect. While great care was taken to emphasize that the probes were provocations to think about the design of social VR, rather than early prototypes of a final system, the Technology Explorers' comments after experiencing the probes often suggested that they viewed the probes as prototypes. This temptation to view a technology probe as a prototype is well acknowledged in the literature (Boehner, Vertesi, Sengers, \& Dourish, 2007; Gaver et al., 2004; Madden, Cadet-James, Atkinson, \& Watkin Lui, 2014). However, we feel that our approach to the probes, constructing them as social VR software rather than more abstract or playful design evocations, added to the temptation to confuse the probes' purpose. If employing this technique in the future, we would consider more carefully how we could explicitly differentiate the technology probes from the intended final technology or application.

\subsection{Conclusion}

In this chapter, we have highlighted the ways in which the combined use of Action Research and Technology Probes helped to guide our work with the Technology Explorers during the first stage of our research project. We have also described and reflected upon the techniques employed to deal with the diverse levels of technical understanding evident in the groups and to help build knowledge about the use of VR and virtual avatars for social participation. During the workshop series, the Technology Explorers progressed from having no prior knowledge of VR or virtual avatars, to seasoned users and critics of these emerging technologies. We believe that the techniques employed during the workshop series helped to foster an environment where this development could occur and importantly also provided the time and support necessary to ensure that all the Technology Explorers could make a contribution. We, as a research team, have also benefited tremendously by being involved with the Technology Explorers and believe the techniques employed during the workshops helped us to better understand the challenges and opportunities present in developing social VR applications. We hope that the discussion of our experiences will be of value to all those considering designing and evaluating emerging technologies for older adults. 


\subsection{References}

Anderson-Hanley, C., Snyder, A. L., Nimon, J. P., \& Arciero, P. J. (2011). Social facilitation in virtual realityenhanced exercise: Competitiveness moderates exercise effort of older adults. Clinical Interventions in Aging, 6(1), 275-280. http://doi.org/10.2147/CIA.S25337

Baker, S., Warburton, J., Hodgkin, S., \& Pascal, J. (2017). The supportive network: Rural disadvantaged older people and ICT. Ageing and Society, 37(6), 1291-1309. http://doi.org/10.1017/S0144686X16000350

Baker, S., Waycott, J., Pedell, S., Hoang, T., \& Ozanne, E. (2016). Older people and social participation: From touch screens to virtual realities. Proceedings of the International Symposium on Interactive Technology and Ageing Populations - ITAP '16. http://doi.org/10.1145/2996267.2996271

Bisson, E., Contant, B., Sveistrup, H., \& Lajoie., Y. (2007). Functional Balance and Dual-Task Reaction Times in Older Adults Are Improved by Virtual Reality and Biofeedback Training. CyberPsychology \& Behavior, 10(1), 16-23. http://doi.org/10.1089/cpb.2006.9997

Boehner, K., Vertesi, J., Sengers, P., \& Dourish, P. (2007). How HCI Interprets the Probes. Proceedings of the SIGCHI Conference on Human Factors in Computing Systems (CHI'07), 1077-1086. http://doi.org/10.1145/1240624.1240789

Brand, J. E., \& Todhunter, S. (2015). Digital Australia Report 2016, 27. Retrieved from http://www.igea.net/wp-content/uploads/2015/07/Digital-Australia-2016-DA16-Final.pdf

Brewer, R., \& Piper, A. M. (2016). “Tell It Like It Really Is.” Proceedings of the 2016 CHI Conference on Human Factors in Computing Systems - CHI '16 (Vol. 150, pp. 5529-5542). New York, New York, USA: ACM Press. http://doi.org/10.1145/2858036.2858379

Brydon-Miller, M., Greenwood, D., \& Maguire, P. (2003). Why Action Research? Action Research, 1(1), 9-28. http://doi.org/10.1177/14767503030011002

Carrasco, E., Epelde, G., Moreno, A., Ortiz, A., Garcia, I., Buiza, C., ... Arruti, A. (2008). Natural Interaction between Avatars and Persons with Alzheimer's Disease. In K. Miesenberger, J. Klaus, W. Zagler, \& A. Karshmer (Eds.), Computers Helping People with Special Needs (Vol. 5105, pp. 38-45). Berlin, Heidelberg: Springer Berlin Heidelberg. http://doi.org/10.1007/978-3-540-70540-6_5

Castilla, D., Garcia-Palacios, A., Bretón-López, J., Miralles, I., Baños, R. M., Etchemendy, E., ... Botella, C. (2013). Process of design and usability evaluation of a telepsychology web and virtual reality system for the elderly: Butler. International Journal of Human-Computer Studies, 71(3), 350-362. http://doi.org/10.1016/j.ijhcs.2012.10.017

Cheong, W. L., Jung, Y., \& Theng, Y.-L. (2011). Avatar: A Virtual Face for the Elderly. Proceedings of the 10th International Conference on Virtual Reality Continuum and Its Applications in Industry - VRCAI'11 (p. 491). New York, New York, USA: ACM Press. http://doi.org/10.1145/2087756.2087850

Cyarto, E. V, Batchelor, F., Baker, S., \& Dow, B. (2016). Active ageing with avatars. In Proceedings of the 28th Australian Conference on Computer-Human Interaction - OzCHI '16 (pp. 302-309). New York, New York, USA: ACM Press. http://doi.org/10.1145/3010915.3010944

Flynn, D., van Schaik, P., Blackman, T., Femcott, C., Hobbs, B., \& Calderon, C. (2003). Developing a virtual reality-based methodology for people with dementia: a feasibility study. Cyberpsychology \& Behavior : The Impact of the Internet, Multimedia and Virtual Reality on Behavior and Society, 6(6), 591-611. http://doi.org/10.1089/109493103322725379

Gaver, W., Boucher, A., Pennington, S., \& Walker, B. (2004). Cultural probes and the value of uncertainty. Interactions - Funology, 11(5), 53-56. http://doi.org/10.1145/1015530.1015555

Gaver, W., \& Dunne, A. (1999). Projected realities. Proceedings of the SIGCHI Conference on Human Factors in Computing Systems CHI '99, 600-607. http://doi.org/10.1145/302979.303168

Harley, D., \& Fitzpatrick, G. (2009). YouTube and Intergenerational Communication: The case of Geriatric 1927. Universal Access in the Information Society, 8(1), 1-26.

Hill, R., Betts, L. R., \& Gardner, S. E. (2015). Older adults' experiences and perceptions of digital technology: (Dis)empowerment, wellbeing, and inclusion. Computers in Human Behavior, 48, 415-423. http://doi.org/10.1016/j.chb.2015.01.062 
Hutchinson, H., Bederson, B. B., Druin, A., Plaisant, C., Mackay, W. E., Evans, H., ... Eiderbäck, B. (2003). Technology probes: inspiring design for and with families. Proceedings of the SIGCHI Conference on Human Factors in Computing Systems (CHI '03), (5), 17-24. http://doi.org/10.1145/642611.642616

Lewin, K. (1946). Action Research and Minority Problems. Journal of Social Issues, 2(4), 34-46. http://doi.org/10.1111/j.1540-4560.1946.tb02295.x

Light, A., Howland, K., Hamilton, T., \& Harley, D. A. (2017). The Meaning of Place in Supporting Sociality. Proceedings of the 2017 Conference on Designing Interactive Systems - DIS '17, 2, 1141-1152. http://doi.org/10.1145/3064663.3064728

Madden, D., Cadet-James, Y., Atkinson, I., \& Watkin Lui, F. (2014). Probes and prototypes: a participatory action research approach to codesign. CoDesign, 10, 31-45. http://doi.org/http://dx.doi.org/10.1080/15710882.2014.881884

Miller, K. J., Adair, B. S., Pearce, A. J., Said, C. M., Ozanne, E., \& Morris, M. M. (2014). Effectiveness and feasibility of virtual reality and gaming system use at home by older adults for enabling physical activity to improve health-related domains: a systematic review. Age and Ageing, 43(2), 188-195. http://doi.org/10.1093/ageing/aft194

Mirelman, A., Maidan, I., Herman, T., Deutsch, J. E., Giladi, N., \& Hausdorff, J. M. (2011). Virtual reality for gait training: Can it induce motor learning to enhance complex walking and reduce fall risk in patients with Parkinson's disease? Journals of Gerontology - Series A Biological Sciences and Medical Sciences, 66 A(2), 234-240. http://doi.org/10.1093/gerona/glq201

Mirelman, A., Rochester, L., Reelick, M., Nieuwhof, F., Pelosin, E., Abbruzzese, G., ... Hausdorff, J. M. (2013). V-TIME: a treadmill training program augmented by virtual reality to decrease fall risk in older adults: study design of a randomized controlled trial. BMC Neurology, 13(1), 15. http://doi.org/10.1186/1471-2377-13-15

Moyle, W., Jones, C., Dwan, T., \& Petrovich, T. (2017). Effectiveness of a Virtual Reality Forest on People With Dementia: A Mixed Methods Pilot Study. The Gerontologist, Advance online publication. http://doi.org/10.1093/geront/gnw270

Ozanne, J. L., \& Saatcioglu, B. (2008). Participatory Action Research. Journal of Consumer Research, 35(3), 423-439. http://doi.org/10.1086/586911

Reason, P., \& Bradbury, H. (2008). The Sage handbook of action research : participative inquiry and practice. Thousand Oaks, Calif.: SAGE Publications.

Rice, M., Koh, R., Lui, Q., He, Q., Wan, M., Yeo, V., ... Tan, W. P. (2013). Comparing avatar game representation preferences across three age groups. CHI'13 Extended Abstracts on Human Factors in Computing Systems on - CHI EA '13, 1161. http://doi.org/10.1145/2468356.2468564

Rogers, Y., Paay, J., Brereton, M., Vaisutis, K. L., Marsden, G., \& Vetere, F. (2014). Never Too Old: Engaging Retired People Inventing the Future with MaKey MaKey. Proceedings of the 32Nd Annual ACM Conference on Human Factors in Computing Systems (pp. 3913-3922). New York, NY, USA: ACM. http://doi.org/10.1145/2556288.2557184

Schultheis, M. T., \& Rizzo, A. a. (2001). The application of virtual reality technology in rehabilitation. Rehabilitation Psychology, 46(3), 296-311. http://doi.org/10.1037/0090-5550.46.3.296

Singh, A., Johnson, A., Alnizami, H., \& Gilbert, J. E. (2011). The Potential Benefits of Multi-Modal Social Interaction on the Web for Senior Users. J. Comput. Sci. Coll., 27(2), 135-141.

Vines, J., Pritchard, G., Wright, P., Olivier, P., \& Brittain, K. (2015). An Age-Old Problem. ACM Transactions on Computer-Human Interaction, 22(1), 1-27. http://doi.org/10.1145/2696867

Vutborg, R., Kjeldskov, J., Pedell, S., \& Vetere, F. (2010). Family storytelling for grandparents and grandchildren living apart. In Proceedings of the 6th Nordic Conference on Human-Computer Interaction Extending Boundaries - NordiCHI '10 (pp. 531-540). http://doi.org/10.1145/1868914.1868974

Warburton, J. (2014). Ageing and social policy in Australia. In S. Harper \& K. Hamblin (Eds.), International Handbook of Ageing and Public Policy (pp. 301-317). Cheltenham: Edward Elgar.

Warburton, J., Cowan, S., Winterton, R., \& Hodgkin, S. (2014). Building Social Inclusion for Rural Older People Using Information and Communication Technologies: Perspectives of Rural Practitioners. Australian Social Work, 67(4), 479-494. http://doi.org/10.1080/0312407X.2013.834064 
Waycott, J., Vetere, F., Pedell, S., Kulik, L., Ozanne, E., Gruner, A., \& Downs, J. (2013). Older adults as digital content producers. Proceedings of the SIGCHI Conference on Human Factors in Computing Systems - CHI '13 (p. 39). New York, New York, USA: ACM Press. http://doi.org/10.1145/2470654.2470662

Waycott, J., Vetere, F., Pedell, S., Morgans, A., Ozanne, E., \& Kulik, L. (2016). Not For Me : Older Adults Choosing Not to Participate in a Social Isolation Intervention. Proceedings of the 2016 CHI Conference on Human Factors in Computing Systems - CHI '16. New York, New York, USA: ACM Press. http://doi.org/ $10.1145 / 2858036.2858458$

Winterton, R., \& Warburton, J. (2011). Does place matter? Reviewing the experience of disadvantage for older people in rural Australia. Rural Society, 20(2), 187-197. http://doi.org/10.5172/rsj.20.2.187

Zancanaro, M., Gabrielli, S., Jameson, A., Leonardi, C., Not, E., \& Pianesi, F. (2013). Virtual Helper or Virtual Card Player? Contrasting Responses of Older Users. In Your Virtual Butler. The Making-of: LNCS 7407 (pp. 70-78). FBK, Trento, Italy: Springer-Verlag. http://doi.org/10.1007/978-3-642-37346-6_7

Zuber-Skerritt, O., \& Perry, C. (2002). Action research within organisations and university thesis writing. The Learning Organization, 9(4), 171-179. http://doi.org/10.1108/09696470210428895 



\section{University Library}

\section{- M M N E R VA A gateway to Melbourne's research publications}

Minerva Access is the Institutional Repository of The University of Melbourne

Author/s:

Baker, S;Waycott, J;Vetere, F;Hoang, T

Title:

The Technology Explorers: Partnering with Older Adults to Engage with Virtual Reality and Virtual Avatars

Date:

2019

Citation:

Baker, S., Waycott, J., Vetere, F. \& Hoang, T. (2019). The Technology Explorers: Partnering with Older Adults to Engage with Virtual Reality and Virtual Avatars. Neves, BB (Ed.). Vetere, F (Ed.). Ageing and Digital Technology: Designing and Evaluating Emerging Technologies for Older Adults, (1), pp.231-246. Springer Singapore.

Persistent Link:

http://hdl.handle.net/11343/241888 